\begin{tabular}{|c|l|}
\hline Title & Polarization-discriminated spectra of a fiber-microsphere system \\
\hline Author(s) & Konishi, Hidenori; Fujiwara, Hideki; Takeuchi, Shigeki; Sasaki, Keiji \\
\hline Citation & $\begin{array}{l}\text { A pplied Physics Letters, 89(12), 121107 } \\
\text { https://doi.org/10.1063/2355473 }\end{array}$ \\
\hline Issue Date & 2006-09-19 \\
\hline Doc URL & http://hdl.handle.net/2115/33855 \\
\hline Rights & $\begin{array}{l}\text { Copyright 2006 A merican Institute of Physics. This article may be downloaded for personal use only. Any other use } \\
\text { requires prior permission of the author and the A merican Institute of Physics. }\end{array}$ \\
\hline Type & article \\
\hline File Information & A pplPhysLett_89_121107.pdf \\
\hline
\end{tabular}

Instructions for use 


\title{
Polarization-discriminated spectra of a fiber-microsphere system
}

\author{
Hidenori Konishi, Hideki Fujiwara, Shigeki Takeuchi, ${ }^{a}$ and Keiji Sasaki \\ Research Institute for Electronic Science, Hokkaido University, Sapporo 060-0812, Japan
}

(Received 14 May 2006; accepted 5 August 2006; published online 19 September 2006)

\begin{abstract}
Polarization-discriminated spectra of a fiber-microsphere system were acquired. The authors have succeeded in developing a single-mode tapered fiber capable of maintaining the polarization of the probe beam. The spectra acquired from this system discriminated between transverse electric and transverse magnetic modes. (C) 2006 American Institute of Physics. [DOI: 10.1063/1.2355473]
\end{abstract}

Microsphere resonators ${ }^{1-3}$ coupled with a tapered optical fiber have been attracting considerable attention recently as ideal cavity systems having ultrahigh $Q$ factors and single spatial mode input-outputs. After the pioneering demonstrations of coupling between a silica glass microsphere and a tapered fiber, ${ }^{4,5}$ applications for lasers, ${ }^{6-8}$ biosensors, ${ }^{9}$ and the observation of the Fano effect ${ }^{10}$ have been reported.

However, the transverse electric (TE) and transverse magnetic (TM) modes in microsphere resonators coupled with a tapered fiber have not been discriminated in previous reports. ${ }^{11}$ This may be due to the technical difficulty of preserving the linear polarization of light passing through a tapered fiber. Very recently, Guan and Vollmer reported polarized transmission spectra from a fiber-microsphere system. ${ }^{12}$ However, in their experiment, the polarization of the probe beam was not preserved in the fiber and the TE and TM transmission modes were not discriminated.

In this letter, we report the acquisition of polarizationdiscriminated spectra from a fiber-microsphere system. We succeeded in developing a single-mode tapered fiber that preserves the polarization of the probe beam. In addition, the spectra obtained from the fiber-microsphere system discriminated the TE and TM modes. We believe that this technique will allow fiber-microsphere systems to be used in polarization-dependent optical devices including quantum information devices. ${ }^{13-15}$ For example, it is now possible to measure the nonlinear phase shift of the light (photon) in the coupled polarization mode using the noncoupled polarization mode as a reference using the fiber-microsphere system.

A tapered fiber was prepared from a fused-silica singlemode optical fiber for transmission at a wavelength of $780 \mathrm{~nm}$ (Newport, F-SE). The fiber was heated using a ceramic heater and stretched at both ends. In order to realize adiabatic propagation of light through the tapered region, the curvature of the taper profile should be as small as possible. Using trial and error, we found that the best speed to stretch the fiber was $150 \mu \mathrm{m} / \mathrm{s}$ at a heater temperature of $1600{ }^{\circ} \mathrm{C}$. Using these fabrication conditions, the diameter of the tapered region was found to be approximately $2 \mu \mathrm{m}$ by optical microscope observation, and the length of the whole tapered region was $66 \mathrm{~mm}$. The transmittance of the fiber was consistently high (about 83\%) over the measured wavelength region (from 771 to $789 \mathrm{~nm}$ ), suggesting that the tapered fiber had single-mode transmission for these wavelengths. Another fiber fabricated under the same conditions had more than $90 \%$ transmittance over the same wavelength region. A

${ }^{a)}$ Electronic mail: takeuchi@es.hokudai.ac.jp microsphere having a stem was fabricated by melting the tip of a fused-silica fiber using a carbon dioxide laser beam. ${ }^{16}$ The diameter of the sphere was measured using an optical microscope and found to be $178 \mu \mathrm{m}$.

The experimental setup we used is shown in Fig. 1. A linearly polarized laser beam from a tunable external-cavity laser diode (New Focus TLB-6312) having a linewidth of $300 \mathrm{kHz}$ and whose absolute wavelength was calibrated using the $\mathrm{Rb}$ gas absorption lines was used as the probe beam. The polarization of the probe beam was rotated using a half wave plate (HWP1) and the beam was then coupled into a single-mode fiber. A microsphere having a stem was connected to the tapered region of the fiber. When we measured the polarization transmission properties of the tapered fiber, another half wave plate (HWP4) and a polarizing beam splitter (PBS3) were inserted at the output of the fiber coupler in order to analyze the polarization state. The probe beam was then detected by a photodiode (Hamamatsu Photonics, S1226-8BQ). The output of the photodiode was measured using a digital oscilloscope (Tektronix, TDS5034-B).

First, we verified that when linearly polarized light was inputted into the tapered fiber the output obtained was also linearly polarized. Figure 2 shows the HWP4 angle dependent intensity of the probe beams with the polarizations at $\theta^{\prime}=0^{\circ}, 45^{\circ}, 90^{\circ}$, and $135^{\circ}$, where $\theta^{\prime}=\theta-54^{\circ}$. Here, $\theta$ is the angle of polarization before fiber coupler $(\mathrm{FC} 1)$ and is $0^{\circ}$ for horizontally polarized light. The average of the visibilities for the fitting curves was 0.985 . The high visibilities for all the curves indicate that linear polarization having any polarization angle was well preserved in the tapered fiber.

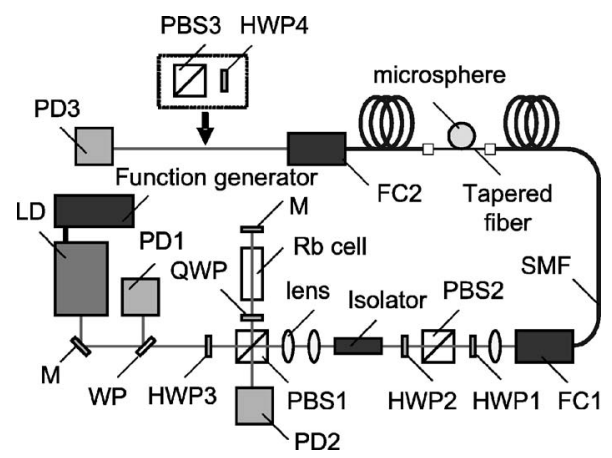

FIG. 1. Experimental setup (top view). LD: tunable laser diode; M: mirror; WP: wedge plate; HWP: half wave plate; QWP: quarter wave plate; PBS: polarizing beam splitter; PD: photodiode; FC: fiber coupler; SMF: singlemode fiber. PBS3 and HWP4 positioned at the output were inserted when the polarization transmission properties of the fiber shown in Fig. 2 were measured. 


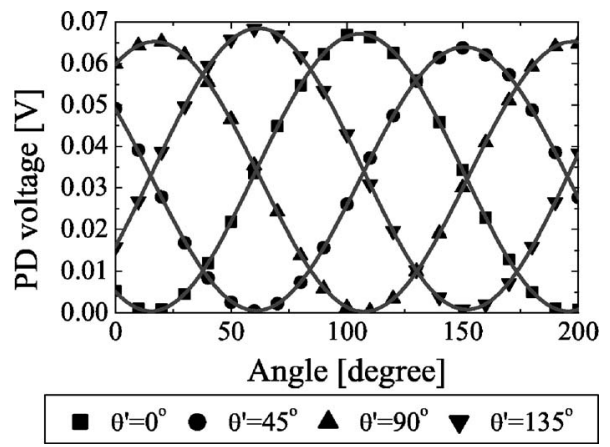

FIG. 2. Polarization-dependent intensity of the output laser power obtained by varying the tilting angle of the linearly polarized probe input light. The experimental data were fitted with sine functions (solid lines) to derive visibilities.

Next, we measured the transmittance spectrum of the tapered fiber in contact with the microsphere by scanning the frequency of the probe beam. The frequency of the probe beam was scanned by applying a chopping voltage from a function generator to the piezomodule of the laser. The intensity of the pumping laser was $80 \mu \mathrm{W}$ measured after HWP1, which was low enough to ensure that there was negligible influence of thermal expansion of the sphere on the spectra obtained. The spectra acquired for $\theta^{\prime}=0^{\circ}, 45^{\circ}$, and $90^{\circ}$, are shown in Fig. 3. We verified that the spectrum acquired at $\theta^{\prime}=180^{\circ}$, which has the same polarization as the configuration with $\theta^{\prime}=0^{\circ}$, exactly the same as the spectrum acquired at $\theta^{\prime}=0^{\circ}$, confirming that the drifts of the peaks were well compensated by the calibration performed using the $\mathrm{Rb}$ absorption spectrum in our experiment. We labeled the resonant peaks at $\theta^{\prime}=0^{\circ}$ (lower one) as An for $n$ $=1-21$, and those at $\theta^{\prime}=90^{\circ}$ (upper one) as $\mathrm{B} n$ with $n$ $=1-15$.

First of all, the differences in the spectra acquired at $\theta^{\prime}$ $=0^{\circ}$ and $\theta^{\prime}=90^{\circ}$ are noteworthy. Among the resonant peaks at $\theta^{\prime}=0^{\circ}$, many strong peaks (A7, A8, A16-A21) are not clearly visible in the spectrum acquired at $\theta^{\prime}=90^{\circ}$. Similarly, many strong peaks (B3, B6, B10, B11, B14, and B15) in the spectrum acquired at $\theta^{\prime}=90^{\circ}$ vanish in the one acquired at $\theta^{\prime}=0^{\circ}$. Furthermore, most of the resonant peaks ( $\mathrm{A} n$ and $\mathrm{B} n$ ) both in the spectrum acquired at $\theta^{\prime}=0^{\circ}$ and the one acquired at $\theta^{\prime}=90^{\circ}$ are found in the spectrum acquired at $\theta^{\prime}=45^{\circ}$.

Figure 4 shows the normalized depth $d$ of the dips (A16 and B11) as a function of the input polarization $\theta^{\prime}$. The normalized depth $d$ is defined by $d=(1-T) /\left(1-T_{\min }\right)$, where $T$ is the transmittance at the dips and $T_{\min }$ is the observed minimum value of $T$ for the dips. The experimental data are well fitted by sinusoidal curves, which have almost opposite phases to each other. ${ }^{17}$ Other strong peaks we mentioned in

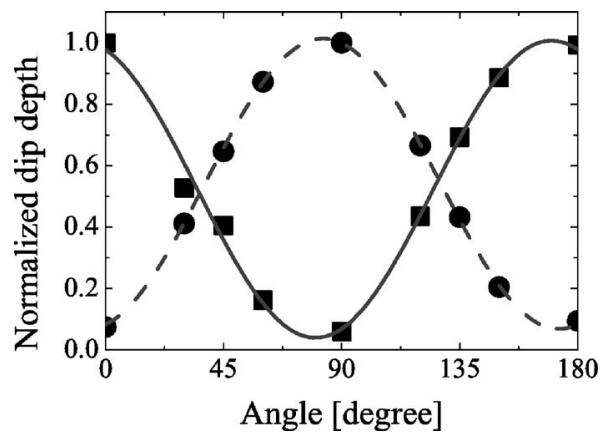

FIG. 4. Normalized depth of the dips, A16 (square) and B11 (circle) as a function of the input polarization. The experimental data were fitted with sinusoidal curves.

the previous paragraph also showed the same behavior.

These experimental results suggest two facts. Firstly, the resonant peaks $\mathrm{A} n$ and $\mathrm{B} n$ correspond to modes having orthogonal polarizations, i.e., TE and TM modes, in the microsphere resonator. Secondly, the probe beam fields, whose polarizations were $\theta^{\prime}=0^{\circ}$ and $\theta^{\prime}=90^{\circ}$ before the $\mathrm{FC} 1$, were also linearly polarized in the tapered region. We think that two of the peaks which appear in both the spectra acquired at $\theta^{\prime}=0^{\circ}$ and $\theta^{\prime}=90^{\circ}$, i.e., $\mathrm{A} 3$ and $\mathrm{B} 2$, are accidentally degenerate in energy.

In order to test these conjectures, we tried to ascertain the polarization of the probe beam in the tapered region by monitoring the polarization of the scattered light through Rayleigh scattering process. The tapered fiber above was observed through a polarizing filter using a microscope equipped with a highly sensitive charge-coupled device camera (Roper Scientific, Cascade512B). We focused our interests in the small dim light spots. ${ }^{18}$ We found that the intensity of those pointlike "dim" light points is maximum when the polarization of the probe beam $\theta^{\prime}$ was $90^{\circ}$ and decreased as the $\theta^{\prime}$ decreased. The intensity was almost 0 at $\theta^{\prime}=0^{\circ}$. The polarization of those scattered light was perpendicular to the light propagating direction (tapered fiber). We confirmed that all the dim light points behaved similarly for ten different places. When we regard those dim lights as Rayleigh scattered light, those experimental results are consistent when we assume that the probe beam in the tapered fiber was almost horizontally polarized at $\theta^{\prime}=90^{\circ}$ and almost vertical at $\theta^{\prime}$ $=0^{\circ}$. The microsphere was touching the side of the tapered fiber. As a result, we conclude that the resonant peaks $\mathrm{A} n$ and $\mathrm{B} n$ correspond to the TE modes and TM modes, respectively.

In conclusion, we have observed polarizationdiscriminated spectra from a fiber-microsphere system. We have succeeded in developing a single-mode tapered fiber

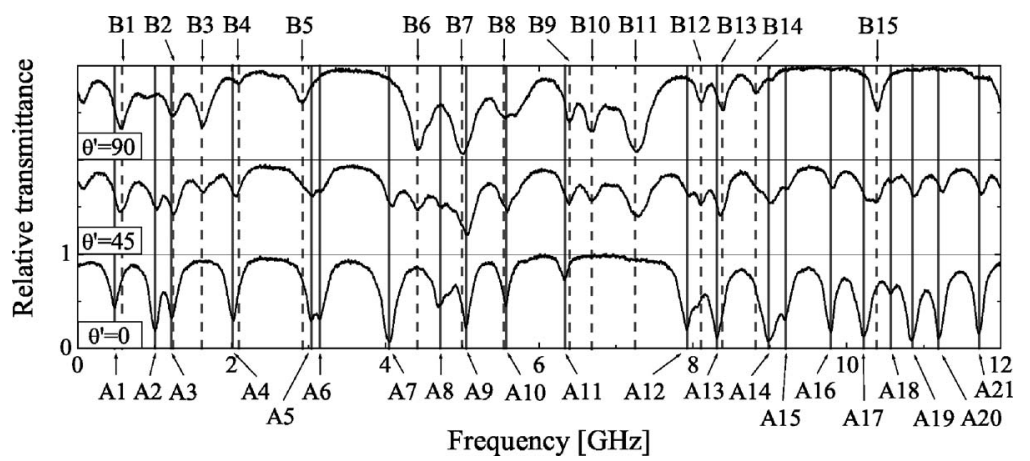

FIG. 3. Transmission spectra of a tapered fiber coupled with a microsphere resonator. Appropriate offsets were added to the data with $\theta=45^{\circ}$ and $\theta=90^{\circ}$ for the sake of clarity. 
which preserves the polarization of the probe beam, and the spectra acquired from this system discriminated between the TE and TM modes. In the future, we intend to determine the polarization-discriminated resonant peaks by comparing experimentally acquired spectra from smaller microspheres with spectra calculated using Mie scattering theory.

The authors would like to thank H. Takashima and A. Chiba for his helpful comments. This study was supported in part by the Ministry of Internal Affairs and Communication (MIC) Strategic Information and Communications R\&D Promotion Programme (SCOPE), the Japan Science Promotion Society (JSPS, No. 17684021), the Japan Science and Technology Corporation (JST) Core Research for Evolutional Science and Technology (CREST) project, and the 21st Century COE Program.

${ }^{1}$ R. K. Chang and A. J. Campillo, Optical Processes in Microcavities (World Scientific, Singapore, 1996).

${ }^{2}$ H. Fujiwara and K. Sasaki, J. Appl. Phys. 86, 2385 (1999).

${ }^{3}$ H. Fujiwara, K. Sasaki, and H. Masuhara, ChemPhysChem 6, 2410 (2005).

${ }^{4}$ A. Serpengüzel, S. Arnold, G. Griffel, and J. A. Lock, J. Opt. Soc. Am. B 14, 790 (1997).

${ }^{5}$ M. Cai and K. Vahala, Opt. Lett. 25, 260 (2000).

${ }^{6}$ M. Cai and K. Vahala, Opt. Lett. 26, 884 (2001).
${ }^{7}$ K. Sasagawa, Z. Yonezawa, R. Iwai, J. Ohta, and M. Nunoshita, Appl. Phys. Lett. 85, 4325 (2004).

${ }^{8}$ A. A. Savchenkov, A. B. Matsko, D. Strekalov, M. Mohageg, V. S. Ilchenko, and L. Maleki, Phys. Rev. Lett. 93, 243905 (2004).

${ }^{9}$ M. Noto, M. Khoshsima, D. Keng, I. Teraoka, V. Kolchenko, and S. Arnold, Appl. Phys. Lett. 87, 223901 (2005).

${ }^{10}$ A. Chiba, H. Fujiwara, J. Hotta, S. Takeuchi, and K. Sasaki, Appl. Phys. Lett. 86, 261106 (2005).

${ }^{11}$ Polarization selective coupling with a prism coupler has been reported by S. Götzinger, L. de S. Menezes, O. Benson, D. V. Talapin, N. Gaponik, H. Weller, A. L. Rogach, and V. Sandoghdar, J. Opt. B: Quantum Semiclassical Opt. 6, 154 (2004).

${ }^{12}$ G. Guan and F. Vollmer, Appl. Phys. Lett. 86, 121115 (2005).

${ }^{13}$ H. F. Hofmann, K. Kojima, S. Takeuchi, and K. Sasaki, J. Opt. B: Quantum Semiclassical Opt. 5, 218 (2003).

${ }^{14}$ K. Kojima, H. F. Hofmann, S. Takeuchi, and K. Sasaki, Phys. Rev. A 68, 013803 (2003).

${ }^{15}$ R. Okamoto, S. Takeuchi, and K. Sasaki, Phys. Rev. Lett. 95, 210506 (2005).

${ }^{16}$ A. Chiba, H. Fujiwara, J. Hotta, S. Takeuchi, and K. Sasaki, Jpn. J. Appl. Phys., Part 1 43, 6138 (2004).

${ }^{17}$ Note that the minimum (maximum) position of the curve for A16 (B11) is at $\theta^{\prime}=80^{\circ}$, and slightly different from $\theta^{\prime}=90^{\circ}$, indicating that peaks $\mathrm{A} n$, may be extincted more perfectly at $\theta^{\prime}=80^{\circ}$.

${ }^{18}$ Some larger and brighter light spots with complicated shape were also observed. We think that those lights were due to scatterers whose sizes were comparable to or larger than the wavelength of the probe beam inside the fiber. 